\title{
Self-organizing Maps and Ancient Documents
}

\author{
Eddie Smigiel ${ }^{1}$, Abdel Belaid ${ }^{2}$, and Hatem Hamza ${ }^{3}$ \\ ${ }^{1}$ LICIA-INSA de Strasbourg \\ 24, Bd de la Victoire F-67084 Strasbourg Cedex \\ eddie.smigiel@insa-strasbourg.fr \\ ${ }^{2}$ LORIA, Université Nancy 2 \\ Campus Scientifique - BP 239 \\ 54506 Vandoeuvre-lès-Nancy Cedex \\ abelaid@loria.fr \\ ${ }^{3}$ LORIA, CPE Lyon \\ domaine scientifique de la Doua.Bat 3083 \\ bd du 11 Novembre 1918.BP 2077. \\ 69616 Villeurbane cedex \\ hamza@loria.fr
}

\begin{abstract}
This paper presents how Self-Organizing Maps and especially Kohonen maps can be applied to digital images of ancient collections in the perspective of valorization and diffusion. As an illustration, a scheme of transparency reduction of the digitized Gutenberg Bible is presented. In this two steps method, the Kohonen map is trained to generate a set of test vectors that will train in a supervised manner a classical feed-forward network. The testing step consists then in classifying each pixel into one class out of four by feeding directly the feed forward network. The pixels belonging to the transparency class are then removed.
\end{abstract}

\section{Introduction}

To assure longer conservation and worldwide diffusion, libraries have dashed into vast programs of digitalization of their collections [1].

One of the most emblematic document of human history, the Gutenberg Bible that started the history of printing in the occidental world has been worldwide available through the world wide web for a few years. In the case of an ancient and rare document as the Gutenberg Bible, digitalization aims to provide a wider access to material which needs to be protected from too frequent handling in the perspective of worldwide diffusion but also improved conditions for scholar studies, for instance, comparison of two copies. The digital images can be compared more easily than the physical books themselves. The possibility of comparing two copies is valuable because no two copies of the Gutenberg Bible are exactly identical [2].

After digitalization, no matter how well this stage has been done [3], it is necessary or desirable to apply a wide variety of image processing techniques to valorize this digital document. Among others, image restoration, compression, segmentation and then, character recognition may be envisaged for a wide variety of applications. For 
this kind of tasks, Artificial Neural Networks (ANN) have already proven their abilities [4].

In this paper, we propose to apply Self-Organizing Maps (SOM) to the digitized Gutenberg Bible. There remain four vellum full exemplars throughout the world, one being conserved in Göttingen. The Niedersächsische Staats und Universitätsbibliothek Göttingen has achieved its digitalization and the whole Bible (1282 pages) has been available on a web site ${ }^{1}$ since June 2000. However, probably due to the lighting needed for digitalization, the pages present an important show-through effect. The reverse page information appears on the right-hand image and degrades the general aspect and sometimes the readability of the text.

In the perspective of ancient collections valorization, image restoration is an important topic. Among others, transparency reduction may be interesting in the perspective of this emblematic document worldwide diffusion and valorization. After transparency reduction, one may see facsimiles looking much more like the original pages would appear with good light conditions in the Göttingen University library.

\section{Color Image Classification Using Self-organizing Maps}

Image color clustering by means of self-organizing maps has been proposed in various works to achieve different goals, for instance, segmentation and compression [5-10]. This paragraph is a very brief reminder of so-called self-organizing maps also known as Kohonen maps, applied on the particular case where the input vector is formed by the three RGB components of one image pixel. Complete developments may be found in $[11,12]$.

The competitive layer consists in $\mathrm{S}$ neurons ( $\mathrm{S}$ being chosen by the user depending on the problem to solve). A three components weight vector is associated to each neuron and represents the RGB components of this neuron. The competitive layer computes the distances between the current pixel and each neuron. The one which is the closest to the current pixel wins and is the unique whose weight vector gets modified according to the Kohonen learning rule. Thus, the neuron whose weight vector was closest to the current pixel is updated to be even closer. The result is that the winning neuron is more likely to win the competition if a similar pixel is presented later, and less likely to win when a very different pixel is presented. As more and more pixels are presented, each neuron in the layer adjusts its weights towards a group of pixels. Eventually, if there are enough neurons, every cluster of similar pixels will have a neuron that outputs 1 when a pixel in the cluster is presented, while outputting a 0 at all other times. Thus, the competitive network learns to categorize the pixels of the given image.

Self-organizing feature maps (SOM or SOFM) learn to classify input vectors according to how they are grouped in the input space. They differ from competitive layers in that neighboring neurons in the self-organizing map learn to recognize neighboring sections of the input space. Thus, self-organizing maps learn both the

1 http://www.gutenbergdigital.de/ 
distribution (as do competitive layers) and topology of the input vectors they are trained on. The neurons in the layer of a SOFM are arranged originally in physical positions according to a topology function. As for the competitive layer, the SOFM computes the distance between the input pixel and each neuron of the map. Every winning neuron updates then its weights and, in addition to that, its neighbours update their weights too. This feature of the SOFM is responsible for the network ability to learn the topology of the input space.

Whereas both the competitive layer and the SOFM use unsupervised learning, Learning Vector Quantization networks (LVQ) is a method that uses supervised learning. LVQ networks classify input vectors into target classes by using a competitive layer to find subclasses of input vectors, and then combining them into the target classes by means of a linear network trained for instance with backpropagation. LVQ networks can classify any set of input vectors, not just linearly separable sets of input vectors. The only requirement is that the competitive layer must have enough neurons, and each class must be assigned enough competitive neurons. To ensure that each class is assigned an appropriate amount of competitive neurons, it is important that the target vectors used to initialize the LVQ network have the same distributions of targets as the training data the network is trained on. If this is done, target classes with more vectors will be the union of more subclasses.

\section{Application on the Digitized Gutenberg Bible}

\subsection{General Principle}

The general principle consists in classifying each pixel of the page to be processed in one class out of four: background, right-hand text, colored letters and transparency. This classification then leads to practical applications as transparency reduction or compression which will be described in the following paragraphs. One may notice that we have used RGB components in our work. However, it might be interesting to apply the same ideas with other color frames as HSV, YIQ or CIE L*a*b* that could be more suited.

The method consists first in selecting a characteristic sample of the page to be processed. The sample has not to be too large (in number of pixels) to avoid prohibitive computation time. It has also to include the main features of the collection, such as colored characters, transparency and visible writing. Figure 1 shows an extract of one page of the Gutenberg Bible (folio 11r of the Genesis Book in volume 1). A small window including the reference letter in the upper left corner of the image is used to establish the Kohonen map.

Figure 2 shows the pixel distribution of the preceding sample in the RGB space. Pixels near the origin represent the right-hand text pixels (close to black) on the righthand page; the cloud in the upper right corner represents background pixels. A big cloud just above the one close to the origin with a strong blue component represents the pixels of the blue reference letter. At last, a little diffuse cloud with a strong red component corresponds to the few pixels of the few red-colored letters on the right hand text. 


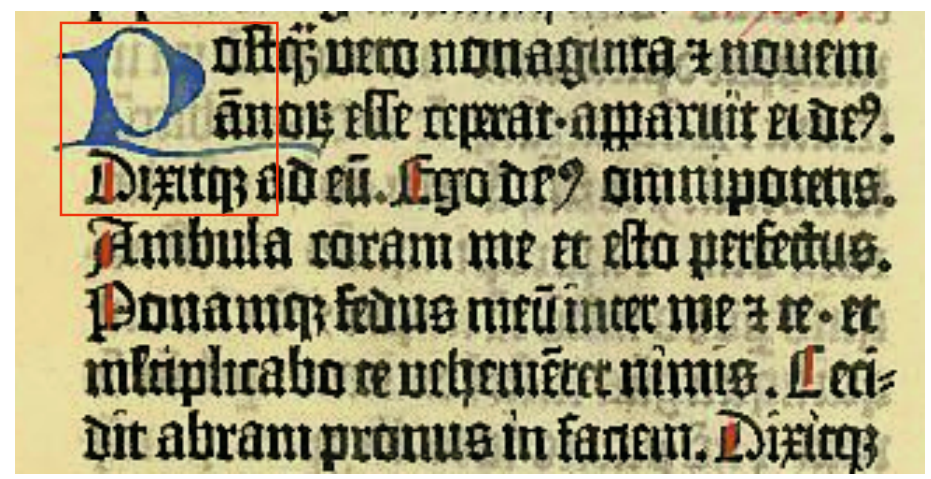

Fig. 1. Extract of the page to be processed (folio 11r of the Genesis Book in Vol. 1). The upper left square represents the training set of the Kohonen map.

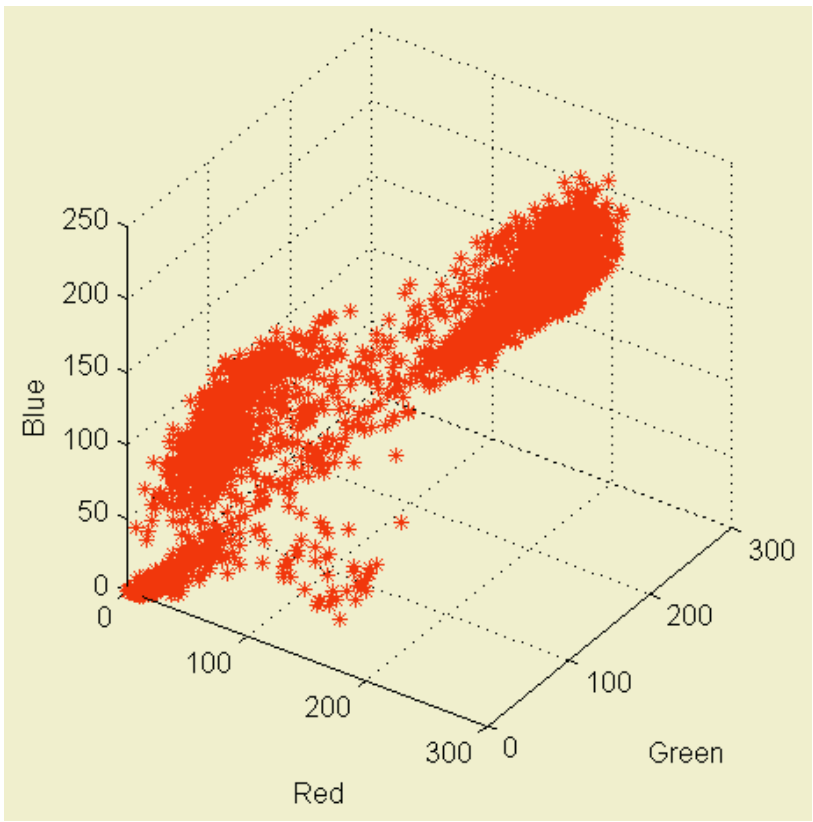

Fig. 2. Pixel distribution of the image sample corresponding to figure 1 in the RGB space.

Figure 3 shows the Kohonen map obtained after training on the small window. It consists in a retangular grid topology of 7 by 7 neurons trained using the euclidean distance and during 50 epochs. The principle characteristics of SOMs appear on this figure: the neurons density depends directly on the number of pixels of the corresponding zone in the input space and the topology is obviously conserved. Note here that the choice of the $7 * 7$ dimension of the Kohonen map is just for visualization clarity and it is not really used for the final experiment. 


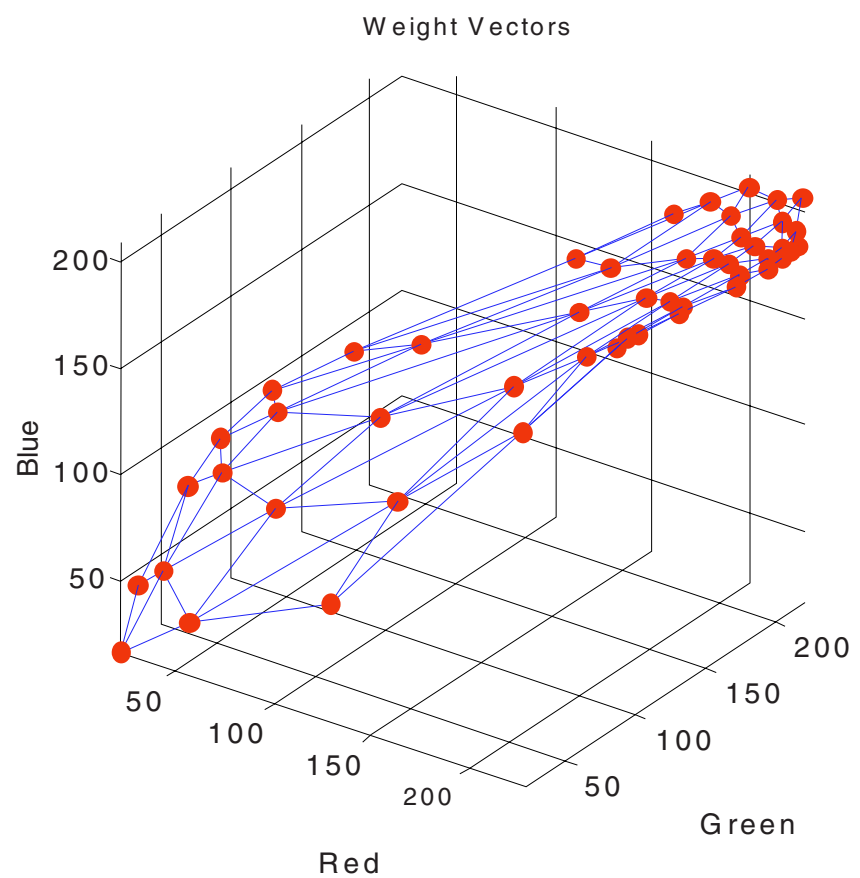

Fig. 3. Kohonen map obtained after a 50 epochs training fed with the pixel distribution of figure 2. The neuron layer has a rectangular topology and is 7 by 7 neurons wide.

\subsection{Transparency Reduction}

As a first idea to apply self-organizing maps to ancient collections, we propose to reduce the show-through effect that degrades the general aspect and the readibility of the digitized pages of the exemplary of the Gutenberg Bible held in Göttingen. One may object that it should be quite easy to find out a few rules on the RGB components to remove or at least to reduce this transparency (for instance, transparency pixels are not contrasted too much and the RGB components should not be very different). These few rules applied pixel by pixel on the whole page would probably achieve a good result. This is not false. In a similar way, other works have produced good algorithms to reduce transparency. There have been a number of methods for mitigating showthrough effects through digital image processing techniques. Naïve methods are based on extensions to binarization techniques for gray-scale document images $[13,14,15]$. To resolve the limitations of the naïve methods, sophisticated methods have been developed on the assumption that images on both sides of the paper sheet are acquired and processed so that the front side image can be compared with the backside image [16]. Correspondence between the two images needs to be established with pixel precision in some way in order to estimate the transmission coefficient of the paper sheet. However, it is difficult to apply this approach to ordinary input devices because solv- 
ing the correspondence problem is intractable due to nonlinear shape distortion of page images. That is why more sophisticated methods have been proposed since $[17,18]$.

However, one should not forget that color clustering may lead to other results beyond transparency reduction (for instance, image compression). One should then consider this particular application (even if it may have some interest in itself) as an illustration of the self-organizing maps applied to color images.

It is possible to classify input pixels into four different classes: background, righthand text, colored letters (like reference letters) and reverse page pixels showing through. This last class is then to be removed from the original page and replaced by background color (the average of the eight last pixels having been detected as background pixels). Our first approach was to use the SOM followed by Kmeans algorithm as done in [19]. This method gives good results with the Gutenberg bible.

However, as the Kmeans algorithm is not reliable in the case of non separable classes (colored letters are not linearly separable as we can see on Fig.2), we have chosen to use SOM and a supervised neural network.

To achieve this classification, we have used a feed-forward network (FFN) (using back-propagation of the error) with one hidden layer (because the four classes are not linearly separable) with 25 neurons in the hidden layer. To train this network which imposes supervised learning, we have used a set of 100 RGB vectors whose components are the RGB components of a Kohonen map of 10 by 10 neurons trained on the image sample corresponding to figure 1. As a supervised learning method, it is necessary to associate to each element of this set of 100 vectors, the corresponding target, that is, the class in which it has to be put in.

Our method has two steps. The first one consists in training the FFN using the output of the SOM which has been trained on the small window of the chosen image. The testing step consists in feeding the FFN with the pixels of the image to be processed.

Thus, the SOM is only used for the purpose of training. In fact, the SOM has the ability to reduce the dimension of the input data ( small window chosen for training). This means that the weights of the neurons obtained after training the SOM represent the most characteristic colors of the input sample. Then, the FFN is trained on an optimal set of colors.

As the chosen sample is representative of the collection to be processed, we can now test our method on a variety of images from this collection. As shown in figure 4, the testing data is given directly to the FFN. Figure 4 shows the principle of our method:

One has to notice that the first network, as a non supervised learning method, does not require to associate any target to the image sample; the dimension of this image sample is then limited by the computation power of the station. On the other hand, the second network, as a supervised learning method, requires to associate to each test vector its corresponding target. That is why we have chosen to use a set of 100 test vetors, this numerical value being reasonable for the entry by hand of the corresponding targets. 


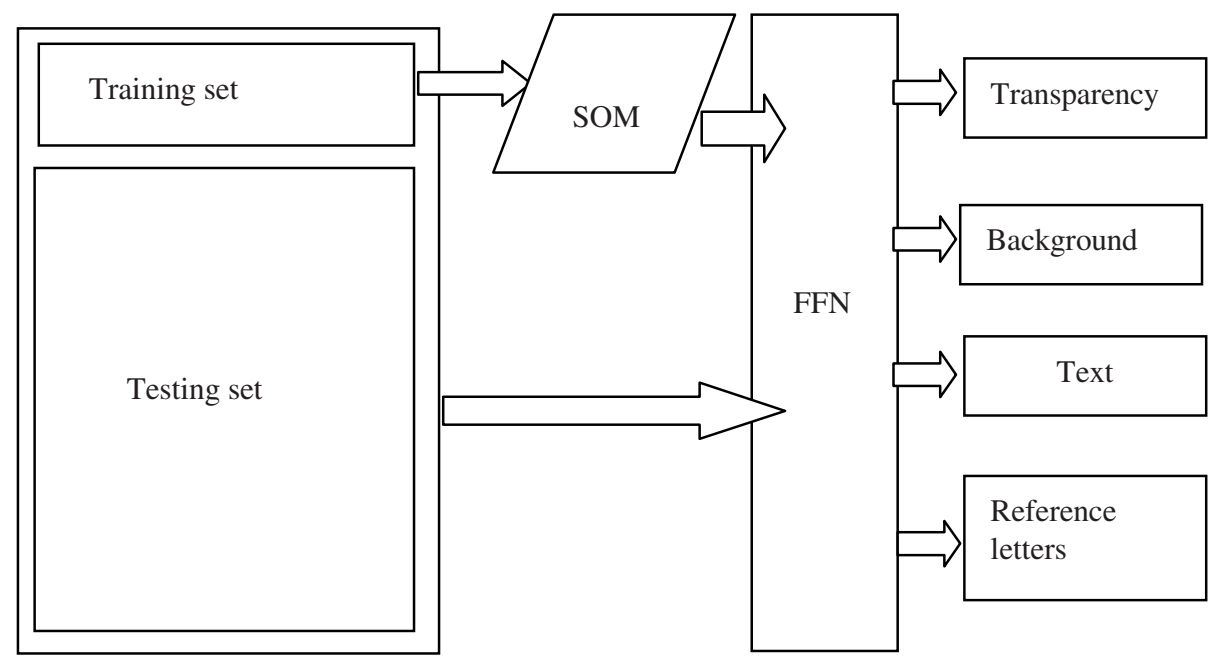

Fig. 4. Principle of the method of pixel classification using SOM and FFN in the training stage and FFN in the testing stage. The testing set can also be another image having the same characteristics as the one from which we have extracted the training data. Note that this figure is directly related to the Gutenberg Bible. For other books or collections, the number of classes in the output of the FFN can be different, but the general principle will be exactly the same.

The number of neurons is guided by two constraints. The first requirement is to have a sufficiently large number of neurons compared to the number of the final classes. For instance, a $2 * 2$ SOM will classify the input image into 4 classes, which would disqualify the use of the FFN. Our experiments show that starting from a $5 * 5$ neurons map, we can separate the different classes. On the other hand, having a very large map would make the association "neurons-classes" heavy without improving the results in a significant way.

Figure 5 shows the result of the transparency reduction applied on the extract of figure 1 . Almost $15 \%$ of the total amount of pixels has been detected as transparency and replaced. This rate may seem to be too large but it is due to the fact that during the feed-forward network training, it has been considered that it would be better to mistake a background pixel for a transparency one, the pixel being replaced by a background one anyway rather than to miss a transparency pixel that would not be removed.

On a qualitative point of view, figure 5 shows that the transparency reduction has been efficient: the text is easier to read, the image much more pleasant to look at. However, when one looks carefully at the obtained image, one can see, a) remaining dark pixels corresponding to transparency pixels that were too close to right-hand text to be correctly classified, b) a character trimming effect: some characters have lost some pixels because they have been wrongly removed, their RGB components being close to the transparency class.

To judge the validity of our approach, we have tested in it on other images different from the one from which we have extracted our training data. 


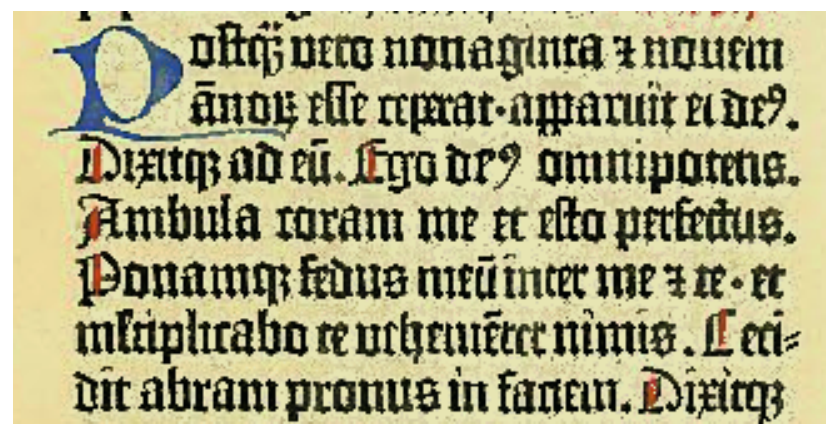

Fig. 5. Result of the transparency reduction applied on teh extract of figure 1 .

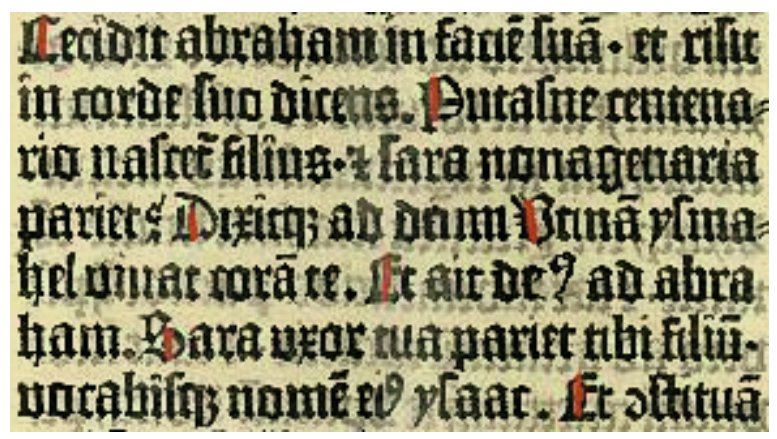

Fig. 6. Test figure. We can see that the transparency is higher in this example.

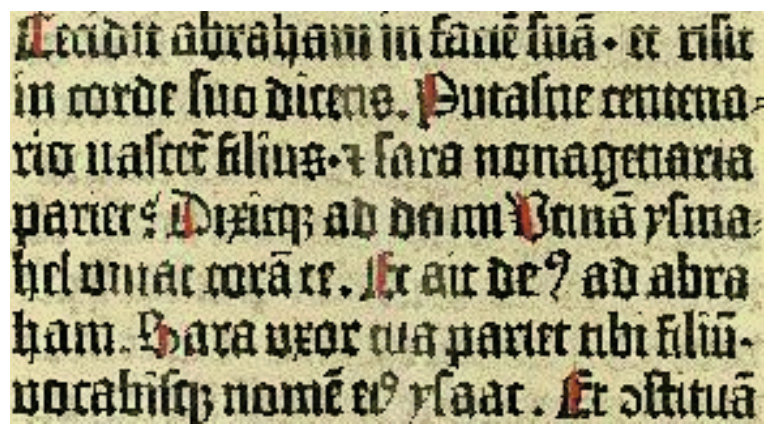

Fig. 7. Result of transparency reduction applied on the extract of Fig.6.

The result has the same characteristics as the first one. Most of the transparency disappeared and we obtain the desired front writing.

Whether simple schemes or more complicated ones like classification by ANN are used, as long as the classification is based on the RGB components of the current pixel, the effect of character trimming described above can not be avoided. A pixel of right-hand text may have RGB components corresponding to a light gray level and 
then be mistaken for a transparency pixel and, similarly, a transparency pixel may be quite dark and be confused with right-hand text. While training, a trade-off has to be found between this two contradictory effects but its efficiency will always be limited. It is also possible to apply rules after classification and before taking decision of removal; for instance, to limit the trimming effect, even though a pixel has been detected as a transparency pixel, the decision not to replace it will be taken if its closest neighbors have been detected as right-hand text.

However, a furher idea would consist in using once again a SOM and a feedforward network whose input vectors would not only be the RGB components of the single current pixel but also the RGB components of its 8 closest neighbors. Thus, the additional rules for removal decision mentionned above would be included in the architecture of the neural network.

\section{Perspectives}

Our method has to be tested on a variety of Document images. There exists a number of images which present a higher transparency and characteristics which are harder to segment. The use of a FFN is then really necessary as it can separate non linearly separable classes of pixels.

As another perspective of this work, we are thinking about an approach which is inspired by the binarization algorithms .In fact, many thresholding techniques use the neighborhood of the pixel to determine its class. These methods can be extended to the classification of the pixels into $\mathrm{N}$ classes instead of 2 classes only. The locally adaptive thresholding techniques seem to be quite adequate for this purpose.

\section{Conclusion}

Self-organizing maps are well suited for color clustering and classification of pixels in any kind of images. By using such a map as the set of test vectors to train a classical feed-forward network, we have described a method of transparency reduction which gives satisfactory results in the field of ancient collections illustrated here by the digitized Gutenberg Bible.

\section{References}

1. J. André, M.-A. Chabin, "Les documents anciens", Document Numérique, volume 3, $n^{\circ} 1-2$, Editions Hermès, 1999

2. B. Agüera y Arcas, "Temporary matrices and elemental punches in Gutenberg's DK type", in "Incunabula and their Readers: Printing, Selling and Using Books in the Fifteenth Century", ed. Kristian Jensen (London: The British Library, 2003).

3. A. R. Kenney, O. Y. Rieger, "Moving theory into practice: Digital imaging for libraries and archives", ISBN: 0-9700225-0-6, April 2000 
4. M. Egmont-Petersen; D. de Ridder, H. Handels, "Image processing with neural networks a review", Pattern Recognition 35 (2002) 2279-2301

5. C. Amerijckx, M. Verleysen, P. Thissen et al., "Image compression by self-organized Kohonen map", IEEE Transactions on Neural Networks 9 (3) (1998) 503-507.

6. G. Hauske, "A self organizing map approach to image quality", Biosystems 40 (1-2) (1997) 93-102.

7. J. Koh, M.S. Suk, S.M. Bhandarkar, "A multilayer self organizing feature map for range image segmentation", Neural Networks 8 (1) (1995) 67-86.

8. T. Kohonen, "Clustering, taxonomy and topological maps of patterns", Proc. 6th IAPR International Conference on Pattern Recognition, München, 1982, pp. 114-128.

9. Y. Zheng, J.F. Greenleaf, J.J. Gisvold, "Reduction of breast biopsies with a modified selforganizing map", IEEE Transactions on Neural Networks 8 (6) (1997)

10. S. Haring, M.A. Viergever, J.N. Kok, "Kohonen networks for multiscale image segmentation", Image and Vision Computing 12 (6) (1994) 339-344.

11. Kohonen, T., Self-Organization and Associative Memory, 2nd Edition, Berlin: SpringerVerlag, 1987.

12. Kohonen, T., Self-Organizing Maps, Second Edition, Berlin: Springer-Verlag, 1997.

13. C.L. Tan, R. Cao, P. Shen, Q. Wang, J. Chee and J. Chang, "Removal of interfering strokes in double-sided document images," in Proc. 5th IEEE Workshop on Applications of Computer Vision (Palm Springs, CA), December 2000, pp. 16-21.

14. R. Cao, C.L. Tan, Q. Wang and P. Shen, "Segmentation and analysis of double-sided handwritten archival documents," in Proc. 4th IAPR International Workshop on Document Analysis Systems (Rio de Janeiro, Brazil), December 2000, pp. 147-158.

15. J. Sauvola and M. Pietikainen, "Adaptative document image binarization," Pattern Recognition, vol. 33, no. 2, 2000, pp. 225-236.

16. Q. Wang and C.L. Tan, "Matching of double-sided document images to remove interference," in Proc. IEEE Computer Society Conf. on Computer Vision and Pattern Recognition (Hawaii), December 2001, vol. I, pp. 1084-1089.

17. G. Sharma, "Show-through cancellation in scans of duplex printed documents," IEEE Trans. Image Processing, vol. 10, no. 5, 2001, pp. 736-754.

18. H. Nishida and T. Suzuki, "A Multiscale Approach to Restoring Scanned Color Document Images with Show-Through Effects", Proceedings of the Seventh International Conference on Document Analysis and Recognition (ICDAR 2003).

19. H. Hamza "Segmentation and restoration of images of old books", LORIA. 